International Journal of Adhesion and Adhesives

April 2018, Volume 82, Pages 184-495

http://dx.doi.org/10.1016/i.ijadhadh.2017.12.003

http://archimer.ifremer.fr/doc/00415/52651/

(c) 2017 Published by Elsevier Ltd.

\title{
A viscoelastic-viscoplastic model to describe creep and strain rate effects on the mechanical behaviour of adhesively-bonded assemblies
}

\author{
Ilioni A. ${ }^{1,{ }^{*}}$, Badulescu C. ${ }^{1}$, Carreere N. ${ }^{1,2}$, Davies Peter ${ }^{2}$, Thevenet D. ${ }^{2}$ \\ ${ }^{1}$ ENSTA Bretagne, IRDL, FRE CNRS 3744, F-29200 Brest, France \\ 2 IFREMER, Marine Structure Laboratory, 29280 Plouzané, France \\ * Corresponding author : A. Ilioni, email address : alin.ilioni@ensta-bretagne.org
}

\begin{abstract}
:
Most of the adhesives used in the marine industry are polymers with a mechanical behaviour which is strongly influenced by the strain rate. Therefore, it is important to predict with accuracy their viscous behaviour. To describe their mechanical behaviour in a bonded joint, a viscoelastic-viscoplastic constitutive law is proposed here. The viscous effects on the elastic behaviour is described using a spectral distribution, which divides the viscous strain as the sum of the strains of several viscous mechanisms, each of them with a different characteristic time and weight. The viscoplastic component of the model permits a better description of the strong non-linear behaviour of the adhesives. The parameters of the constitutive law are obtained using an inverse identification procedure coupled with a finite element model. Two creep tests, in two loading directions, are needed in order to identify the viscoelastic part. The viscoplastic part is identified using monotonic tests. In order to validate the behaviour law and the identification procedure, the adhesive HuntsmanTMAraldite $420 \mathrm{~A} / \mathrm{B}$ has been investigated and modelled. All the experimental tests have been conducted using the modified Arcan device.
\end{abstract}

Keywords : A. Epoxides, D. Mechanical properties of adhesives, D. Creep / mechanical relaxation 


\section{Introduction}

Composite materials have found many applications in naval and other marine structures over the past 50 years. In order to join the composite to other parts of the structure, adhesives represent an interesting solution. They can reduce the weight (avoiding bolts and fasteners) and decrease the stress concentrations that can be catastrophic for the strength of the structures [1].

Different tests can be used to characterize the adhesive directly in the bonded joint as shown by Adams et al. [2]. Single or double lap joints are an interesting solution to this 
problem [3], but due to the geometries of the substrates, they may present high stress singularities near the edges that can cause a premature failure of the joint [4]. Cognard et al. proposed the modified Arcan device in order to characterize the mechanical behaviour of adhesives in bonded joints up to failure. This can be achieved using substrates with special geometries [5] that can reduce the stress concentrations near the edges. This device has been used in this paper in order to characterize the behaviour of the studied adhesive.

Characterizing the mechanical behaviour of the adhesive used in bonded joints is a very important step in order to be able to describe the failure of this kind of structure. Even though these adhesives are essentially polymers with a viscous behaviour, the load transfer between the components of the bonded joint is usually linked to their instantaneous elastic properties [6]. However, the viscous behaviour represents a major characteristic of polymeric adhesive materials. Therefore, the understanding of these phenomena is important in order to accurately predict the failure and the lifetime of bonded structures. The experimental tests performed on bulk specimens show a nonlinear behaviour of adhesives that is very sensitive to the strain rate [7][8]. The viscous response of different structural adhesives was also highlighted under impact loadings in previous studies [9]. All these tests require homogeneous bulk specimens, without any defects, that may be difficult to obtain [10].

Different models have been proposed in the literature in order to predict the mechanical behaviour of epoxy adhesives. In some cases, viscoelastic models $[11][12][13][14]$ can be perfectly adapted to characterize the time-dependent behaviour and to predict the influence of the strain rate. Other authors have proposed elastoplastic models [15][16] or viscoplastic models [17][18][19] which are characterized by a threshold and a permanent strain. While various models of adhesive behaviour have been presented, the originality of the present approach is that the viscoelastic response is treated in two separate parts, hydrostatic and deviatoric. This has allowed different viscoelastic behaviour to be attributed to shear and tensile loading components. In addition, an original identification method for the viscoelastic parameters is presented. 
A viscoplastic contribution is also modeled, derived from the Mahnken-Schlimmer model, but again simplifications have been made to reduce the number of parameters

The article presents a viscoelastic-viscoplastic model that is able to describe the nonlinear behaviour of adhesives over a wide range of strain. The proposed viscoelastic part of the model is able to describe the adhesive behaviour in tensile and shear loading conditions. The viscoelastic strain is decomposed into a number of viscous mechanisms, each of them with a specific relaxation time. The viscous compliance is modelled in a manner that takes into account the effect of the hydrostatic pressure and its evolution to describe the non-linear behaviour as a function of the loading. This approach allows an accurate characterization of time-dependent behaviour. The viscoplastic part of the model is able to take into account the dependency of the elastic threshold as a function of the strain rate. The paper presents the development of the model and its identification procedure based on multi-level creep tests for the normal behaviour and for the shear behaviour using a modified Arcan device. The validation of the model by comparison with experimental results with different ratios of peel/shear stress is finally presented.

\section{Characterization tests and experimental results}

\subsection{Experimental procedure}

\subsubsection{Modified Arcan device}

A bonded joint is an assembly that is very sensitive to testing conditions. There are several important details that should be taken into account in order to be able to perform a valid test. The tests presented have used the modified Arcan device. This device enables different types of loadings to be applied to bonded joint. The positioning angle on the tensile machine defines the state of load that is applied to the adhesive(Fig.1a). Using a uniaxial tensile machine, it is possible to apply shear (Fig.1b) or tensile (Fig.1c) loads on a bonded joint. It is also possible to combine tensile or compression with shear loading (Fig.1d,1e). All these loadings can be applied because of the design of this experimental device. 


\section{ACCEPTED MANUSCRIPT}

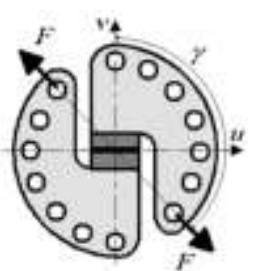

(a)

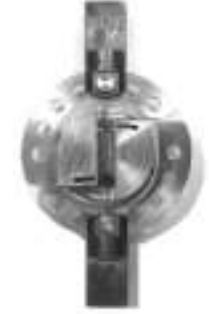

(b)

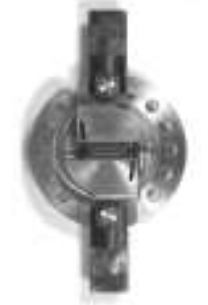

(c)

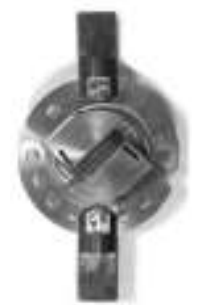

(d)

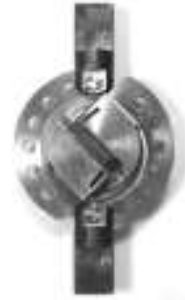

(e)

Figure 1: Different types of Arcan device loading - (a) Positioning angle (b) Shear $\gamma=90^{\circ}$ (c) Tensile $\gamma=0^{\circ}$ (d) Tensile/shear $\gamma=45^{\circ}$ (e) Compression $/$ shear $\gamma=135^{\circ}$

An important aspect to be considered when performing this test is the complex stress state for different loading conditions. Much detailed information concerning this subject can be found in literature [20][21].

\subsubsection{Specimens}

The substrates (Fig.2a) used in the tests are made from aluminium alloy, AW2017. Their geometry was developed and optimized previously as described in the literature [21]. They are provided with spacers (Fig.2a) that are directly machined from a single block of aluminium alloy. These have an important role in order to obtain a correct alignment and keep a constant thickness when bonding a specimen. The results presented in this paper are obtained using a thickness of the bonded joint that is equal to $400 \pm 10 \mu \mathrm{m}$. During the curing cycle the substrates are kept in the final position by screws (Fig.2b).

A constant torque of $2.5 \mathrm{Nm}$ is applied to the screws.

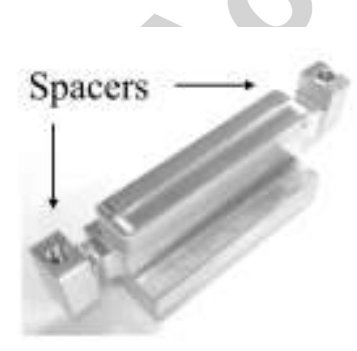

(a)

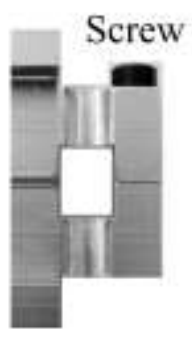

(b)

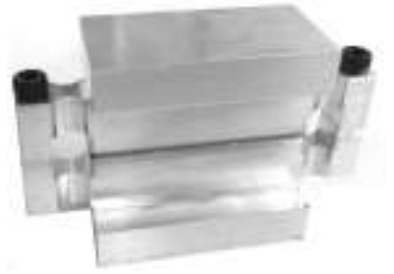

(c)

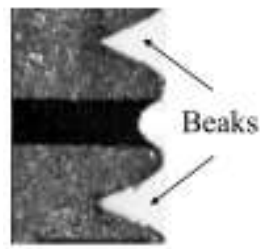

(d)

Figure 2: Modified Arcan specimen - (a) Substrate (b) Screw (c) Specimen (d) Beaks 
Before starting the process of bonding, the bonded surfaces of the specimen $\left(S_{0}=\right.$ $50 \times 9.5 \mathrm{~mm}^{2}$ ) are subjected to a mechanical treatment that involves a sanding process with different tapes of emery cloth $(220$ - 180 grit cloth $)$ in order to ensure a clean surface. The next step is to degrease it using acetone. To ensure an homogeneous joint, the adhesive is applied on the surface of each substrate that will form the specimen. The excess adhesive after the bonding process is cleaned with a spatula.

The final bonded specimen can be seen in Fig.2c. After the curing process, the spacers are removed. An important characteristic of the specimens is the beaks (Fig.2d). They allow the edge effects to be reduced, as shown by Cognard et al. [22] and could lead to a cohesive failure of the assembly.

\subsubsection{Equipment}

To perform the experimental tests, the Arcan device was inserted in a hydraulic tensile testing machine (Instron 1342). The temperature in the testing room is controlled at about $23^{\circ} \mathrm{C}$. The load cell can measure a maximum load of $100 \mathrm{kN}$.

The relative displacement of the substrates is measured using a DIC (digital image correlation) system (Fig.3a) and no other independent measuring system was used in the present study. The specimen is speckled on one face before installing it in the Arcan device (Fig.3b) and the shaded zone represents the area of interest for calculating the displacements. This relative displacement is correlated with the load measured by the tensile machine load cell.

The relative displacement between the two substrates during a test is decomposed into a normal displacement, (ND) and a tangential displacement (TD), (Fig.3c).

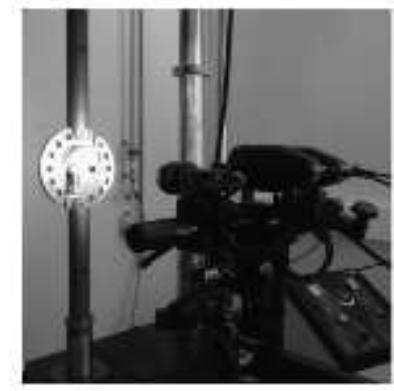

(a)

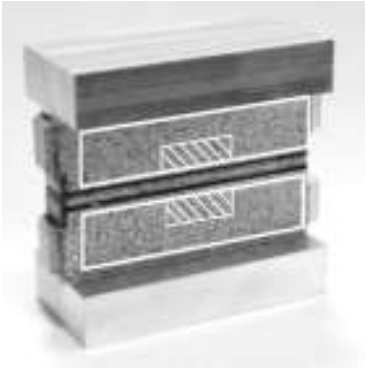

(b)

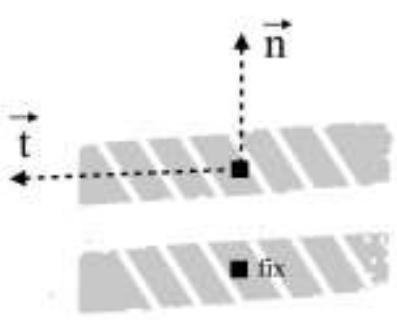

(c)

Figure 3: Measurement of substrates displacements - (a) GOM System (b) Speckled specimen ready for test (c) Digital Image Correlation 


\subsection{Experimental results $E D$ MANUSCRIPT}

The following results were obtained using the Huntsman Araldite 420A/B adhesive. This is a bi-component epoxy. The two components are mixed using a Speedmixer at $2500 \mathrm{rpm}$ for 5 minutes. After the bonding process the specimens are cured for $1 \mathrm{~h} 15$ at $115^{\circ} \mathrm{C}$.

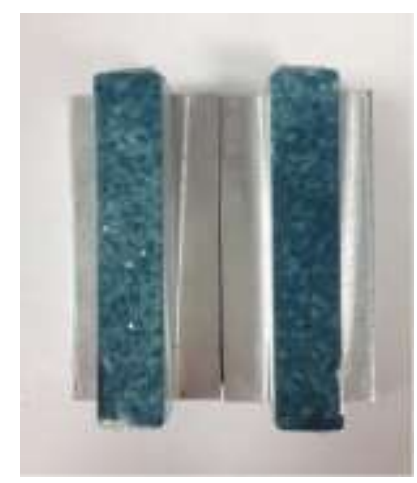

(a)

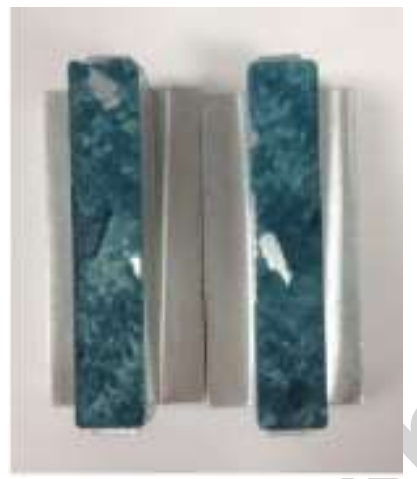

(b)

Figure 4: Cohesive failure in (a) tensile and (b) shear loading

Two types of tests have been performed to characterize the behaviour of this adhesive: monotonic tests in order to characterize the general behaviour of the adhesive up to failure and multi-level creep tests at different loading levels. The latter were performed to highlight the viscoelastic behaviour of the adhesive and to identify the constitutive law that will be presented later in this paper.

It should be noted that in the two cases, tensile and shear loading, a cohesive failure is obtained in most of the tests (Fig.4a,4b). For each type of load, between two and three specimens were tested since low scatter could be observed, particularly in shear loading (Fig.5b). It is also important to note that the relationship between the elastic limits is statistically equivalent for the different loading rates.

\subsubsection{Stress-strain curves representation}

In order to make possible the correlation of the adhesive behaviour with other published results, a stress-strain representation of the force-displacement curves is 
proposed. Hence, a mean stress $\sigma$ was defined as the applied load " $F$ " divided by the bonded are $S_{0}$ (Eq.1):

$$
\bar{\sigma}=\frac{F}{S_{0}}
$$

Moreover, the normal and shear strains $\left(\overline{\varepsilon_{N}}, \overline{\varepsilon_{T}}\right)$, eare defined as in Eq.2:

$$
\overline{\varepsilon_{N}}=\frac{D N}{e} \quad \overline{\varepsilon_{T}}=\frac{D T}{e}
$$

where $D N$ and $D T$ represent the normal and tangential measured displacements and $e$ the thickness of the adhesive layer.

\subsubsection{Monotonic tensile tests}

Using the Arcan, device it was possible to characterize the monotonic behaviour of this adhesive in tensile $\left(\gamma=0^{\circ}\right)$ and shear load $\left(\gamma=90^{\circ}\right)$. For each condition, two loading rates have been used: $0.4 \mathrm{MPa} / \mathrm{s}$ and $4 \mathrm{MPa} / \mathrm{s}$. It is clear for the two cases (tensile and shear), this adhesive has a mechanical behaviour that is influenced by the loading rate, Fig.5a, 5b.

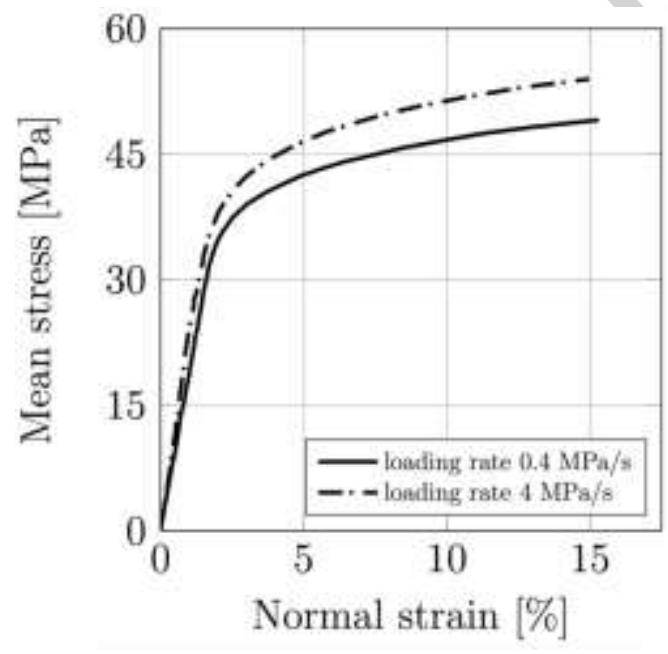

(a)

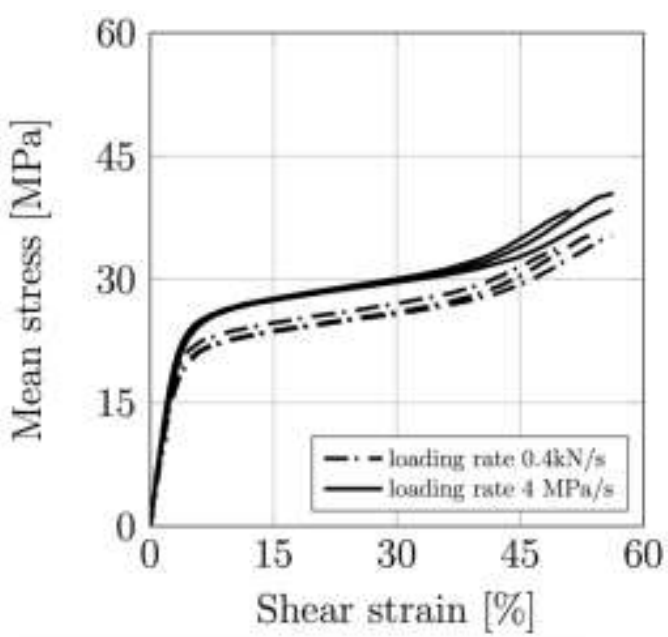

(b)

Figure 5: Monotonic tests: tensile (a) and shear (b)

In tensile tests (Fig.5a), the apparent elastic threshold is increased about $12 \%$ from $28 \mathrm{MPa}$ to $34 \mathrm{MPa}$ when the applied loading rate is multiplied by ten. The failure load is also influenced by the loading rate. The same happens in the shear test. This time it can 
be seen that the apparent elastic threshold is increasing by about $15 \%$ when the loading rate is increased. Some additional combined tensile/shear tests $\left(\gamma=45^{\circ}\right)$ have also been carried out. These tests were used for validation and they will be presented in section 3.4 .

\subsubsection{Multi-level creep tests}

In order to be able to characterize the viscous behaviour of this adhesive, multi-level creep tests have been performed. Only two tests will be used to identify the constitutive law that will be presented next in this paper. The aim was also to highlight any differences between the viscous behaviour under tensile and shear loading conditions

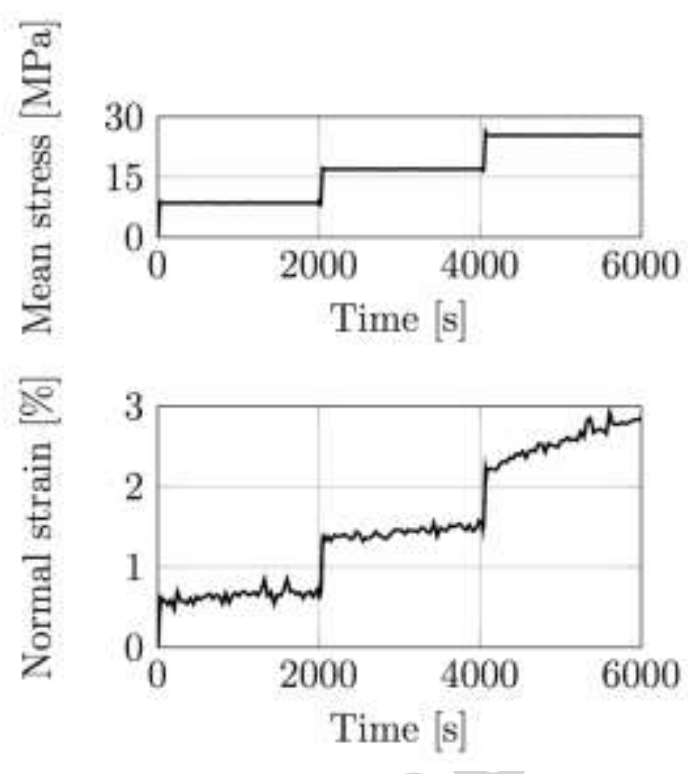

(a)
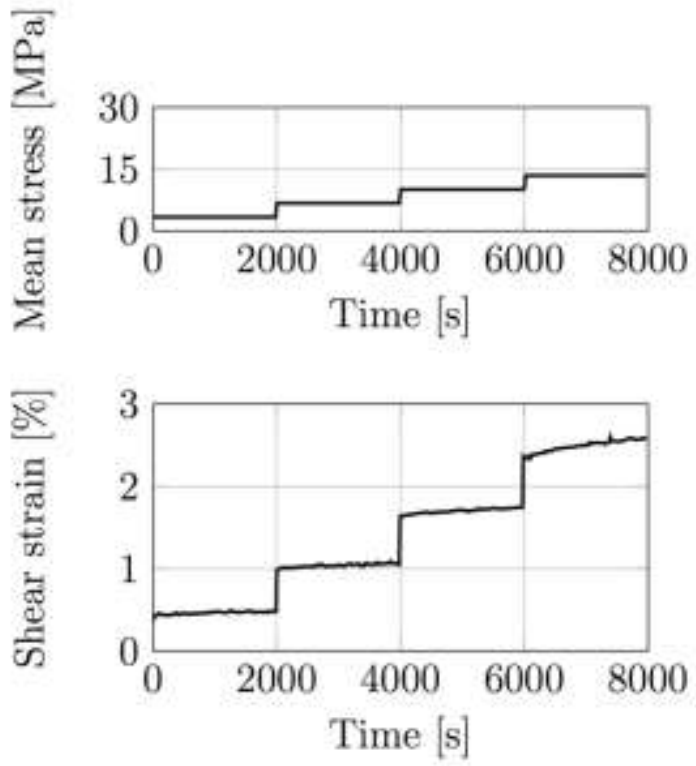

(b)

Figure 6: Multi-level creep tests: tensile (a) and shear (b)

In tension (Fig.6a), three load levels have been applied for a period of 2000 seconds. These values correspond to $25 \%, 50 \%$, and $80 \%$ of the apparent elastic limit at $0.4 \mathrm{MPa} / \mathrm{s}$ loading rate. This loading rate was used to make the passage from one level to another.

Under shear load, (Fig.6b), four load levels have been applied. These levels represent $20 \%, 40 \%, 60 \%, 80 \%$ of the apparent elastic threshold observed in monotonic shear tests for a loading rate of $0.4 \mathrm{MPa} / \mathrm{s}$. Four levels of load have been applied (and not three 
as in tensile loading) since in shear loading condition, a more significant creep response was observed.

The constitutive law was developed by analysing these two results. Based on the work of Badulescu et al. [11] the observed strains $\left({\overline{\varepsilon_{N}}}_{\text {total }}^{i},{\overline{\varepsilon_{T}}}_{\text {total }}^{i}\right)$ at the end of each creep level were divided into two parts, as in Eq.3 and Eq.4, where ${\overline{\varepsilon_{N}}}_{\text {elastic }}^{i}{\overline{\varepsilon_{T}}}^{i}$ elastic represent the strains generated by the applied load at the beginning of each creep level and ${\overline{\varepsilon_{N}}}_{\text {viscous }}^{i},{\overline{\varepsilon_{T}}}_{\text {viscous }}^{i}$ are the strains measured at the end of the creep levels:

$$
\begin{array}{cc}
{\overline{\varepsilon_{N}}}_{\text {total }}^{i}={\overline{\varepsilon_{N}}}_{\text {elastic }}^{i}+{\overline{\varepsilon_{N}}}_{\text {viscous }}^{i} & i=1: 3 \\
{\overline{\varepsilon_{T}}}_{\text {total }}^{i}={\overline{\varepsilon_{T}}}_{\text {elastic }}^{i}+{\overline{\varepsilon_{T}}}_{\text {viscous }}^{i} & i=1: 4
\end{array}
$$

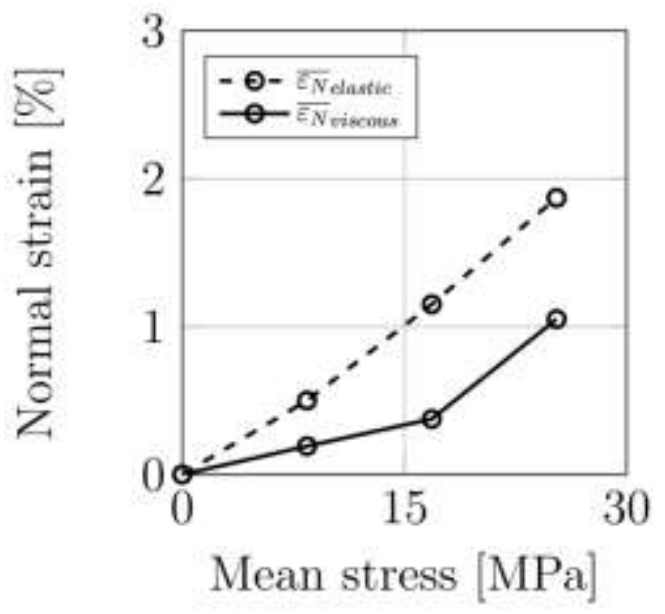

(a)

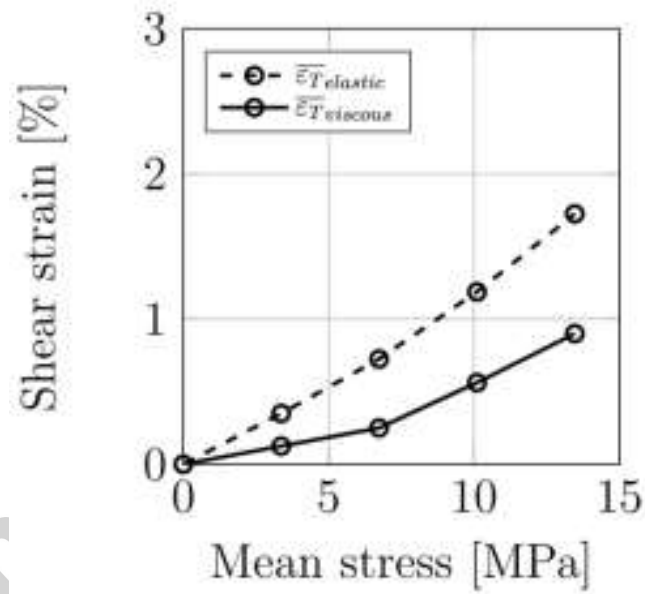

(b)

Figure 7: Evolution of the viscous and elastic displacements at the end of each creep level: (a) tensile and (b) shear

By analysing the results obtained in Fig.7a and Fig.7b it is possible to note that even though the applied load is below the apparent elastic threshold, viscous strains are taking place in both cases: tensile and shear. Another important aspect is that the viscous behaviour is not linear and it depends on the applied load. Moreover, viscous strain observed in shear behaviour is more important than the viscous displacement measured in tensile.

Due to these observations, the non-linear viscoelastic model developed by Badulescu et al [11] was adopted and modified to describe the creep behaviour. The changes made in the model will be presented in the next section. 


\section{Modelling the mechanical behaviour of an epoxy}

\section{adhesive in a bonded joint}

\subsection{Numerical procedure}

In order to describe the mechanical behaviour of the adhesive in the bonded joint it is necessary to develop a realistic FEM model. The model has been developed to be implemented in 3D models that are subjected to complex 3D stress states. In this case, a 2D plane stress analysis would have been sufficient but we were constrained to use a model with one element in the width direction to have all the components of the stress and strain tensors. This approach implies an important reduction in terms of computation time. As has been shown in other studies by Cognard et al. [21], the stress state in the adhesive is not uniform, therefore an inverse identification method will be used in order to identify the parameters of the constitutive law.

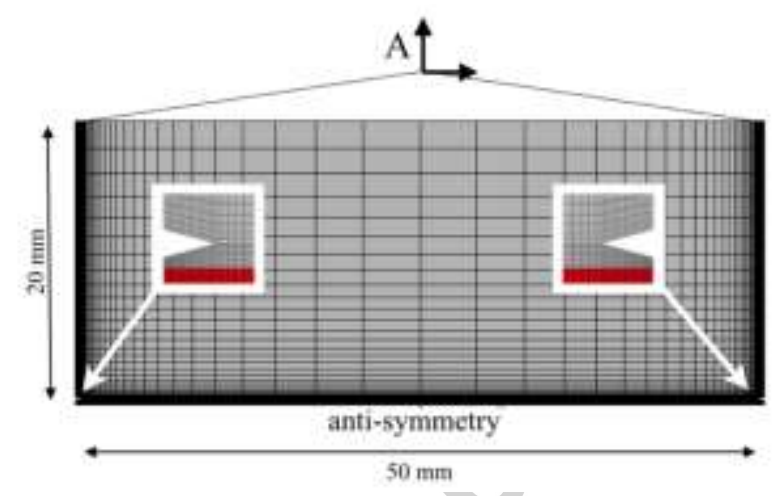

(a)

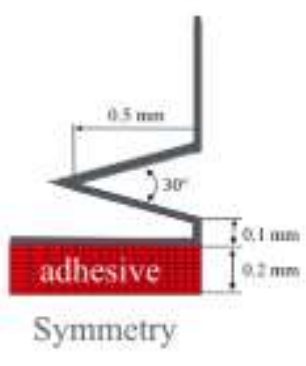

(b)

Figure 8: Finite element model (FEM) of the assembly: (a) the geometry used to model the substrate and the bonded joint, (b) geometry of the beaks

Because of the geometry of the sample, only half of the sample has been modelled. An anti-symmetry boundary condition has been applied in the middle of the bonded joint. The load is computed in the loading point "A" (Fig.8a) and the relative displacement are calculated at the point located in the same zone that the one used for the experimental characterization. Depending on the direction of the applied force, it is possible to obtain the expected stress state (tensile or shear). 
The geometry used for the beaks (Fig.8b) allows the reduction of the stress concentration near the free edge. As can be observed in Fig.8a, the dimensions of the mesh have been decreased near the beaks. A convergence study has been performed in order to determine the right number of elements. The mechanical behaviour of the substrates is assumed to be linear elastic with a Young's modulus equal to 70GPa and a Poisson's ratio of 0.34 . These are the mechanical characteristics of the aluminium alloy used for the substrates.

The bonded joint has a thickness of $0.4 \mathrm{~mm}$. Eight elements have been used to model half of the joint thickness because of the symmetry of the sample. This number has been chosen after a convergence study in order to obtain good results in terms of time and precision. The mechanical behaviour of the adhesives is described using the constitutive law presented in the next section of this paper.

\subsection{Constitutive law}

The final constitutive law used to describe the mechanical behaviour of the adhesive in the bonded joint is based on a viscoelastic-viscoplastic model. As will be shown in the next subsections, the viscoelastic part of the model was not sufficient to describe accurately the mechanical behaviour of the adhesive.

The development of the behaviour law will be presented step by step, starting with a method of identification for the elastic parameters. The viscoelastic part is then described and all the parameters presented. Finally, the viscoplastic part is added to the model in order to obtain the constitutive law.

It is important to note that in each case, the total strain $\varepsilon$ is written as the sum of the different strains: elastic strain $\underline{\varepsilon_{e l}}$, viscoelastic strain $\underline{\varepsilon_{v e}}$, and viscoplastic $\underline{\varepsilon_{v p}}$ (Eq.5).

The stress tensor $\sigma$ will then be written as in $E q .6$, where $\overline{\bar{C}}$ represents the instantaneous elastic stiffness.

$$
\underline{\varepsilon}=\underline{\varepsilon_{e l}}+\underline{\varepsilon_{v e}}+\underline{\varepsilon_{v p}}
$$




\subsubsection{Identification of the elastic parameters}

The elastic parameters of the model (Young's modulus E and Poisson's ratio $v$ ) are the first parameters that have to be identified. It is important to note that in this step the viscoelasticity and the viscoplasticity have no influence on the mechanical response of the model. The experimental curves (load vs displacement), obtained in monotonic tensile and shear Arcan tests, are used to determine an experimental elastic stiffness $(E q .7,8)$ using the measured load $(F)$ and displacements (ND and TD).

$$
\begin{aligned}
& K_{N}=\frac{F}{N D} \\
& K_{T}=\frac{F}{T D}
\end{aligned}
$$

In parallel, using the finite element model, a series of simulations are made using different couples of $(E, v)$. The range of Young's Modulus is chosen between 500MPa and $6000 \mathrm{MPa}$ (with a step of 100MPa), and the Poisson's ratio between 0.2 and 0.45 (with a step of 0.01). These are classical values that can be found in the literature for this kind of adhesive. The numerical stiffness obtained for each couple in both cases (tensile and shear) are compared with the experimental ones.

At the end of each simulation an error is calculated between the numerical and the experimental stiffness. This error is represented in Fig.9a for the tensile loading and in Fig.9b for the shear loading case. The shadowed zone in those figures represents the couples $(E, v)$ that have a minimum error compared to the experimental results. In Fig.9c the couples with the minimal error are superposed in order to find the best couples that fit in both cases (tensile and shear loading). In Table 1 can be found the obtained values for the elastic parameters. 
ACCTable 1:Elastic parameters

\begin{tabular}{ccc} 
Parameters: & E & $v$ \\
\hline Unite & $\mathrm{MPa}$ & {$[-]$} \\
Value & $2000 \pm 50$ & $0.41 \pm 0.01$
\end{tabular}

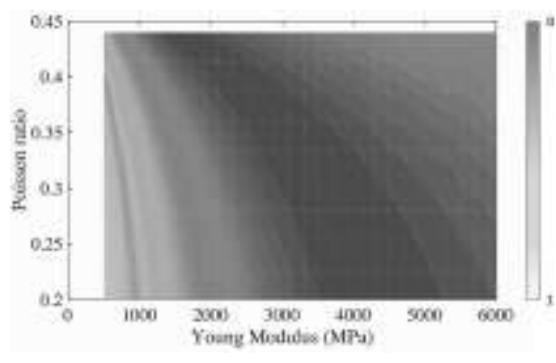

(a)

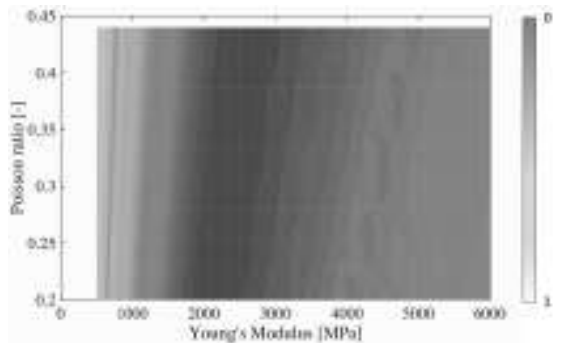

(b)

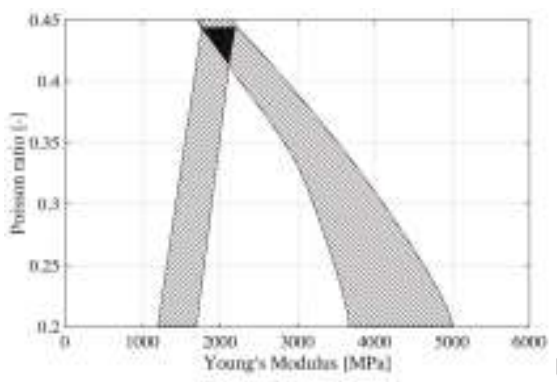

(c)

Figure 9: Calculated error obtained for different couples of $(E, v)$ in (a) tensile and (b) shear behaviour. (c) Intersection of the best couples $(E, v)$

\subsubsection{Modified viscoelastic spectral model}

In a first step, the viscoelastic spectral model has been chosen to model the viscous behaviour of the adhesive. This model has been developed by Maire et al [23] to describe the non-linear behaviour of polymer matrix composites.

In this model, the total strain $\varepsilon$ is equal to the sum of the elastic strain $\underline{\varepsilon_{e l}}$ and the viscous strain $\underline{\varepsilon_{v e}}$, Eq.9. The mechanical behaviour of the adhesive written in the referential of the bonded joint is the following: Eq.10; where $\sigma$ is the stress tensor, $\overline{\bar{C}}$ is the elastic stiffness.

$$
\begin{gathered}
\underline{\varepsilon}=\underline{\varepsilon_{e l}}+\underline{\varepsilon_{v e}} \\
\underline{\sigma}=\underline{\underline{C}}:\left(\underline{\varepsilon}-\underline{\varepsilon_{v e}}\right)
\end{gathered}
$$

The spectral viscoelastic model describes the viscoelastic strain $\underline{\varepsilon_{v e}}$ as the sum of the strains of a fixed number $n_{t}$ of viscous mechanisms $\xi_{i}$. The presented results are obtained with a number of $n_{t}=20$ mechanisms. Each of these mechanisms is defined by a 
relaxation time $\tau_{i}$, a weight $\mu_{i}$ in the total viscous strain and the viscoelastic compliance $S_{R}$, see Eq.11.

$$
\dot{\varepsilon}_{v e}=\sum_{i}^{n_{t}} \dot{\xi}_{l} \quad \dot{\xi}_{\imath}=\frac{1}{\tau_{i}}\left(\mu_{i} \underline{\underline{S_{R}}}: \sigma-\underline{\dot{\xi}_{l}}\right)
$$

The relaxation times are defined as in $E q .12$ where $n_{i} \in\left[n_{1}, n_{2}\right]$ is a value situated between two limits that depend on the material. In the case of an epoxy material, $n_{1}=$ -20 and $n_{2}=30$. This means that the characteristic times are situated between $10^{-9} s$ and $10^{13} \mathrm{~S}$. The mechanisms with high relaxation times have low weight in this case and they are not activated. By decreasing the upper limit and keeping the same number of viscous mechanism, it would be possible to increase the accuracy of the model but it wouldn't be possible to predict the creep behaviour for very long periods of time.

$$
\begin{gathered}
\tau_{i}=\exp \left(n_{i}\right) \\
\overline{\mu_{\iota}}=\frac{1}{n_{0} \sqrt{\pi}} \exp \left(-\left(\frac{n_{i}-n_{c}}{n_{0}}\right)^{2}\right) \quad \text { with } \quad \mu_{i}=\frac{\overline{\mu_{\iota}}}{\sum_{i}^{n_{t}} \overline{\mu_{\iota}}}
\end{gathered}
$$

The weight of each elementary viscous strain depends on two parameters, $n_{0}$ and $n_{c}$ which are introduced in Eq.13. They have to be identified using the creep tests.

These two quantities, the relaxation time and the weight, define the so called spectrum of the model. The form of this spectrum is defined by two parameters $n_{0}$ and $n_{c}$. Each of the points in Fig.10 represents the relaxation time and the weight of a viscous elementary mechanism.

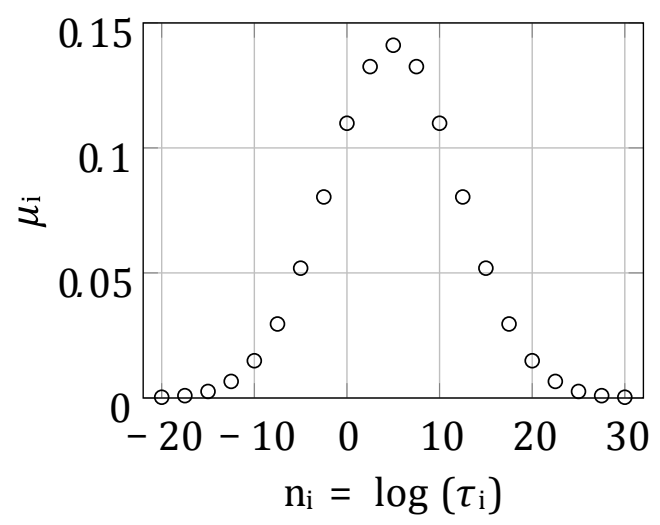

Figure 10: Spectrum definition 
The term $S_{R}$ in Eq.11 represents the viscoelastic compliance. This is a fourth order tensor that has been modified from the previous version of the viscoelastic spectral model [11]. Observing the results obtained in Fig.7, it is possible to note that the adhesive mechanical behaviour depends on the loading conditions. In shear loading, the viscous displacements are more important than in tensile loading. Moreover, a nonlinear evolution of the viscous behaviour can be seen in the two cases. These observations led to the next equation representing the viscoelastic compliance (Eq.14).

$$
\underline{\underline{S_{R}}}=\frac{1}{\left(1-d_{H}\right) a_{H}} \underline{\underline{H_{H}}}+\frac{1}{\left(1-d_{D}\right) a_{D}} \underline{\underline{H_{D}}}
$$

The $S_{R}$ tensor is divided into two parts by the tensors $H_{H}$ and $H_{D}$ which allow the hydrostatic and the deviatoric mechanical behaviour to be defined. The $I$ and $I$ are the identity 2 nd respectively 4 th degree tensors.

$$
\underline{\underline{H_{H}}}=\frac{1}{3} \underline{I} \otimes \underline{I} \quad \underline{\underline{H_{D}}}=\underline{\underline{I}}-\underline{\underline{H_{H}}}
$$

Also, the terms $a_{H}$ and $a_{D}$ in Eq.14 define the viscosity in the hydrostatic and deviatoric directions. These two parameters need to be identified. The non-linearity is introduced using the variables $d_{H}$ and $d_{D} E q .16$ which depend, each of them, on three parameters that need to be identified. The terms $y_{D}^{0}$ and $y_{H}^{0}$ are the threshold of the nonlinear behaviour of the viscosity and $y_{D}^{c}$ and $y_{H} c$ define the kinetics.

$$
d_{H}=d_{H}^{\max }\left(1-\exp \left(\frac{-\left\langle y_{H}-y_{H}^{0}\right\rangle}{y_{H}^{C}}\right)\right) \quad d_{D}=d_{D}^{\max }\left(1-\exp \left(\frac{-\left\langle y_{D}-y_{D}^{0}\right\rangle}{y_{D}^{C}}\right)\right)
$$

The internal forces of the model are $y_{H}$ and $y_{D}, E q .17$. These depend on the hydrostatic and deviatoric stress tensors.

$$
y_{H}=\frac{1}{2 a_{H}}\left(\underline{\sigma}: \underline{\underline{H_{H}}}: \underline{\sigma}\right) \quad y_{D}=\frac{1}{2 a_{D}}\left(\underline{\sigma}: \underline{\underline{H_{D}}}: \underline{\sigma}\right)
$$

This modified viscoelastic spectral model has a total of 10 parameters that need to be identified (Table 2). 


\subsubsection{Identification of viscoelastic parameters RilPT}

The viscoelastic parameters of the model only need two experimental multi-level creep tests in order to identify them (in two loading directions: tensile and shear). The hydrostatic parameters are identified using the tensile multi-level creep test shown in Fig.6a. In the case of the deviatoric parameters, the results obtained in Fig.6b will be used for identification. Because the method of identification is the same in both cases, in the next section, it will be applied only for the deviatoric parameters (shear loading).

a) Identification of the spectrum's parameters: $n_{0}, n_{c}$

The two parameters that define the spectrum are identified using the viscous displacements measured for each creep level. For this, the isochronism principle of the spectral viscoelastic model is used. In order to apply this principle, each creep level should have the same duration. The viscous displacements are normalized using Eq.18, where $\mathrm{j}$ represents the number of the creep level, $j \in\{1,2,3,4\}$ because of the four shear creep levels (Fig.6b). In the same equation, $t_{j_{b e g i n}}$ is the time at the beginning of the $j^{\text {th }}$ level and $t_{j}$ end is the time at the end of the same level. It is also important to mention that the normalized creep curves are not a function of the applied load.

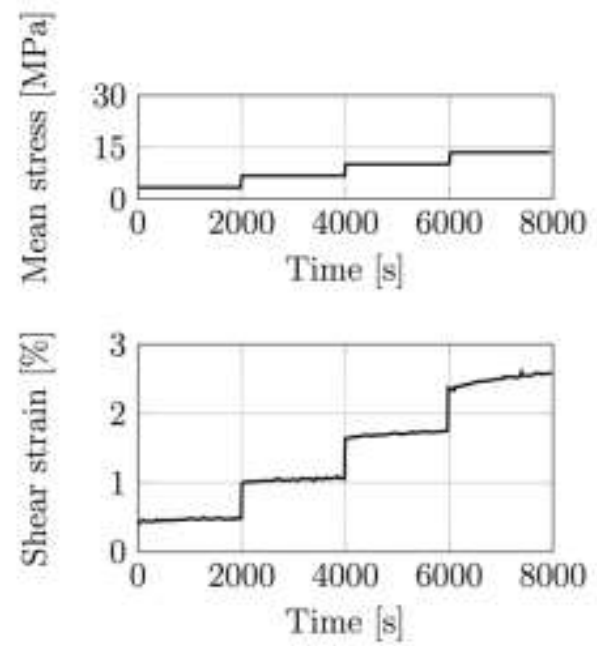

(a)

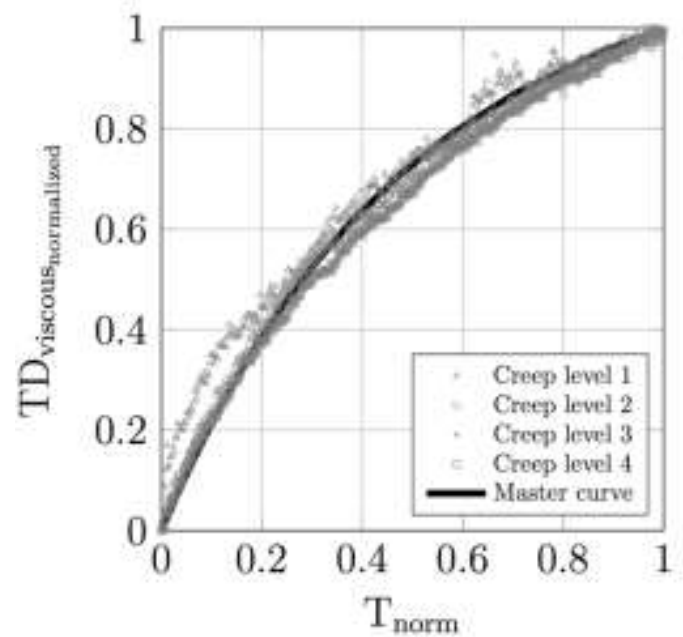

(b)

Figure 11: (a) Multi-level shear creep test $\left(\gamma=90^{\circ}\right)$ used to identify the deviatoric parameters of the viscoelastic model, (b) Isochronism principle 


$$
T D_{\text {viscous }_{\text {normalized }}}^{j}=\frac{D T_{\text {viscous }}^{j}(t)-D T_{\text {viscous }}^{j}\left(t_{\text {begin }}^{j}\right)}{D T_{\text {viscous }}^{j}\left(t_{\text {end }}^{j}\right)-D T_{\text {viscous }}^{j}\left(t_{\text {begin }}^{j}\right)}
$$

In Fig.11b, the normalized viscous displacement can be observed for the four creep levels in Fig.11a. The normalized time for each creep level has been calculated using Eq.19.

$$
T_{\text {norm }}^{j}=\frac{t-t_{\text {begin }}^{j}}{t_{\text {end }}^{j}-t_{\text {begin }}^{j}}
$$

It can be observed in Fig.11b that the isochronism principle is respected for the four creep levels. In this case, these curves can be superposed to a master curve that is a function of the spectrum. The spectrum is then defined by only two parameters that need to be identified: $n_{0}$ and $n_{c}$.

Using the finite element model presented in Section 3.1, it is possible to apply an inverse identification to fit the obtained master curve. For this, a numerical simulation of the first creep level is made. The elastic parameters applied to the model are those identified using the monotonic tests.

$$
\text { Error }=\sum_{1}^{m=200}\left(D T_{\text {viscous }_{\text {normalized }}^{F E}}-D T_{\text {viscous }_{\text {normalized }}}^{\text {EXP }}\right)
$$

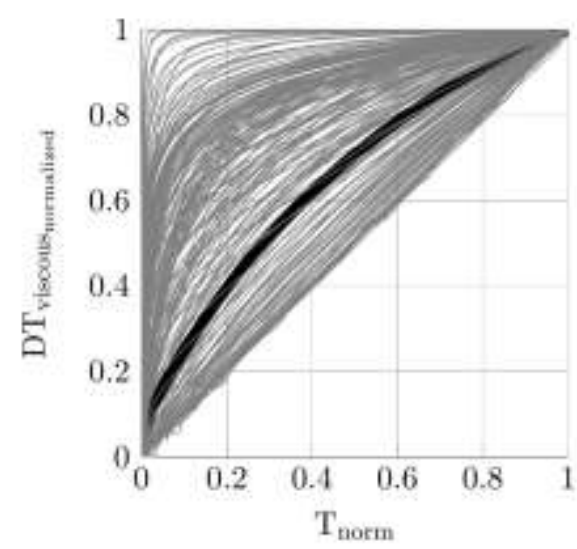

(a)

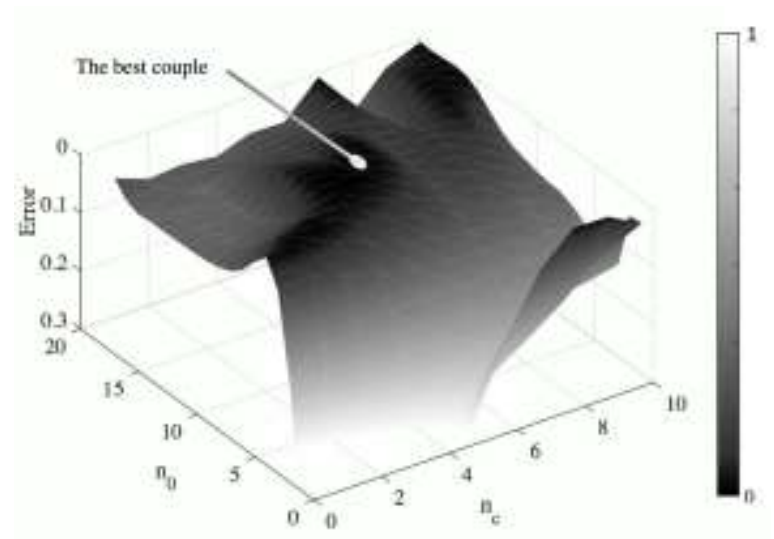

(b)

Figure 12: (a) Normalized viscous displacement for different couples $\left(n_{0}, n_{c}\right)$ (b) Error calculated for different couples $\left(n_{0}, n_{c}\right)$ 
Couples of parameters $\left(n_{0}, \bar{n}_{c}\right)$ have been generated using Matlab software: $n_{c} \in[1$ : $10]$ and $n_{0} \in[1: 20]$ - a total number of 200 combinations. For each couple, a numerical simulation is made. After normalizing the time and the viscous displacements obtained, the results are compared with the master curve created from the experimental curves (Fig.12a). In this stage of the identification process, by normalising the time and the viscous displacement the influence of the rest of the parameters is not taken into account. This process is made automatically using a Python routine.

For each couple an error is calculated using Eq.20. The couple with the closest error to zero is then used. The results are then normalized and they can be represented in an error map, as in Fig.12b. The best couples are those that minimize this error and they have a low influence on the final results.

b) Identification of the linear viscoelastic parameters: $a_{H}, a_{D}$

Once the spectrum is defined, it is now possible to identify the parameters that correspond to the linear viscosity: $a_{H}, a_{D}$. These parameters are identified on the first creep level ( corresponding to the linear viscoelastic behaviour) of the creep tests performed in tensile $\left(a_{H}\right)$ and in shear $\left(a_{D}\right)$, using the inverse identification method and the numerical model. It is important to note that in this step of the identification, the non linear part of the model is not considered. A very high value is fixed to the $y_{H}$ and $y_{D}$ parameters. The results are shown in Fig.13a,13b (curve corresponding to the viscolinear behaviour).
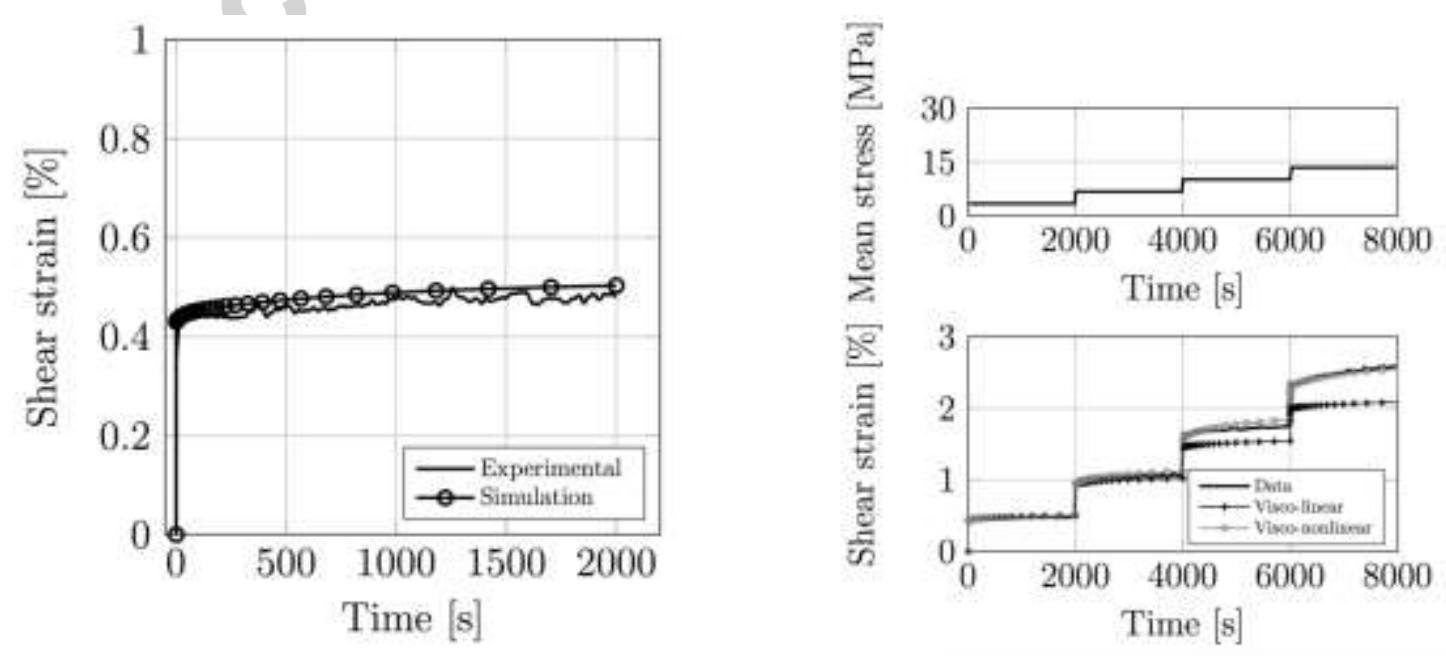
Figure 13: (a) First level of the shear creep test (b) Identification of the viscoelastic model

The identification has been made for the two cases of loading (tensile and shear) using the experimental results obtained in the multi-level creep tests.

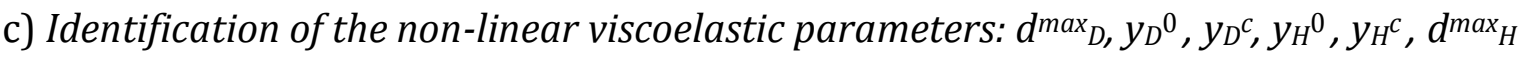

Examining Fig.13b, it can be noted that a linear viscoelastic model is not sufficient to describe correctly the behaviour of the adhesive in the multi-level creep tests. Therefore, the variables $d_{H}$ and $d_{D}$ were introduced in the definition of the compliance tensor.

The threshold between the linear and non-linear viscosity is, in this case, at the end of the second creep level (Fig.13b). Using the FE model, it is possible to evaluate the internal force $y_{D}$ in the middle of the bonded joint where its value is maximal. This value will correspond to the threshold $y_{D}{ }^{0}$. The same approach is applied to find this variable in the case of a tensile creep test.

The last parameters of the model $y_{D}{ }^{c}$ and $d^{\max }{ }_{D}$ and the hydrostatic equivalents are identified using the last two creep levels. These influence the kinetics of the displacements for the last creep levels. It can be seen in Fig.13b that using the non-linear viscosity it is possible to have a good fit between the experimental curve and the numerical results.

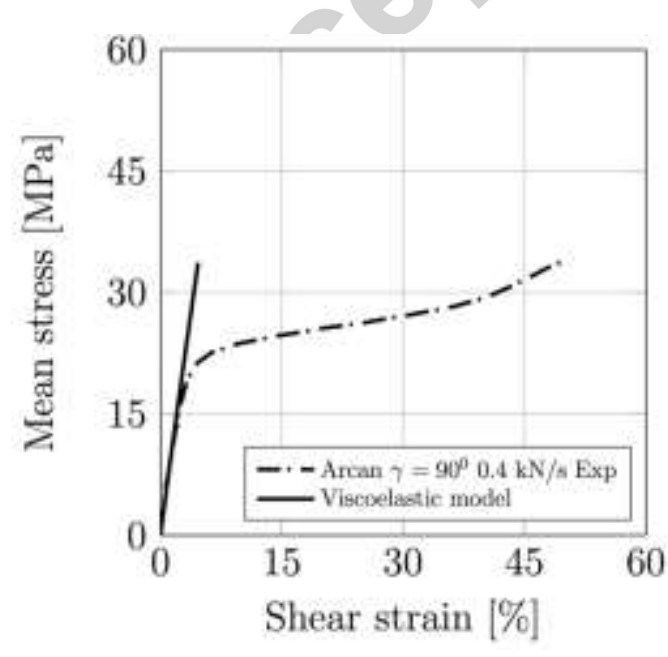

(a)

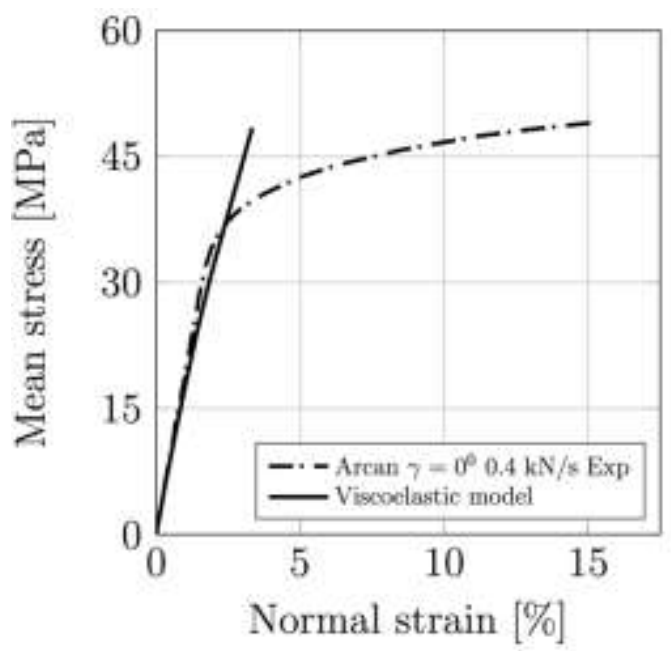

(b)

Figure 14: Viscoelastic model prediction in monotonic (a) shear and (b) tensile tests 


\section{ACCEPTED MANUSCRIPT}

Once the model is validated using the creep tests, it is now possible to evaluate if the model is able to predict the monotonic behaviour.

Fig.14 represents the response of the identified viscoelastic model when simulating a monotonic shear or tensile test with the identified parameters (Table 2). These results are showing that even though the model is describing accurately the creep behaviour, it is not sufficient to predict the mechanical behaviour in monotonic loading. A solution to solve this problem would be to use an elasto-viscoplastic model. But the use of this kind of model would involve to set the elastic limit to a very low value to be able to model the creep behaviour for low loading levels. Thus, in order to predict the mechanical behaviour at high loading-levels, a viscoplastic part have been added in the present constitutive law.

Table 2: Identified viscoelastic parameters

\begin{tabular}{ccccccccccc} 
& \multicolumn{3}{c}{ Spectrum } & \multicolumn{4}{c}{ Hydrostatic } & \multicolumn{4}{c}{ Deviatoric } \\
\hline Parameters & $\mathrm{n}_{0}$ & $\mathrm{n}_{\mathrm{c}}$ & $\mathrm{a}_{\mathrm{H}}$ & $d_{H}^{\max }$ & $y_{H}^{0}$ & $y_{H}^{C}$ & $\mathrm{a}_{\mathrm{D}}$ & $d_{D}^{\text {max }}$ & $y_{D}^{0}$ & $y_{D}^{C}$ \\
\hline Unit & - & - & $\mathrm{MPa}^{-1}$ & - & - & - & $\mathrm{MPa}^{-1}$ & - & - & - \\
Value & 5 & 10 & 4000 & 0.9 & 0.03 & 0.015 & 1500 & 0.6 & 0.01 & 0.02
\end{tabular}

\subsection{Viscoelastic-viscoplastic behaviour}

In the last section, it has been shown that the viscoelastic model is not sufficient to describe correctly the mechanical behaviour of this adhesive. In order to be able to predict the monotonic behaviour, a viscoplastic part has been added to the model. In this case, the total strain $\varepsilon$ will be composed of the elastic strain $\varepsilon_{e l}$, the viscoelastic strain $\varepsilon_{v e}$ and the viscoplastic strain $\varepsilon_{v p}$, as was shown in Eq.5.

The viscoplastic part of the model is inspired by the model proposed by MahnkenSchlimmer et al [15]. In this case, the yield surface is described using a linear relation (Eq.22), between von Mises and hydrostatic stresses. Other forms of this model could be 
found in literature [24][25].This is a simple model that has a few parameters to be identified as it will be shown in the next section.

\subsubsection{Constitutive law for viscoplastic behaviour}

The stress tensor $\sigma$ is decomposed into a deviatoric part $\sigma^{D}$ and a hydrostatic part $\sigma^{H} I$. The von Mises stress $\left(\sigma^{M}\right)$ is then calculated using the Eq.21.

$$
\sigma^{M}=\sqrt{\frac{3}{2} \frac{\sigma^{D}}{\sigma^{D}}} \quad \text { where } \quad \underline{\sigma^{D}}=\underline{\sigma}-\underline{\sigma^{H}} \underline{I} \text { and } \quad \underline{\sigma^{H}}=\frac{1}{3} \operatorname{trace}(\underline{\sigma})
$$

The yield surface of the viscoplastic behaviour is determined using the Eq.22, where $\sigma^{M}$ represents the von Mises stress tensor, $\sigma_{H}$ is the hydrostatic component and R is the hardening parameter. $a_{1}$ is a parameter that defines the shape of the yield function (the slope) in the von Mises - hydrostatic pressure plane.

$$
f=\sigma^{M}+a_{1} \sigma^{H}-R
$$

The Eq.23 represents the evolution of the hardening parameter $R$. The parameters $H$, $Q, b$ influence the monotonic non-linear behaviour of the adhesive and are identified from a pure shear test using the inverse identification method. It is important to note that when those parameters are identified, the viscoelastic part of the model is activated. $R_{0}$ is the elastic threshold.

$$
R=R_{0}+H e_{v c u m}+Q\left(1-\exp \left(-b e_{v c u m}\right)\right)
$$

The hardening is driven by the internal strain variable $e_{v c u m}$ defined in Eq.24. The parameters $K$ and $n$ are identified after all the other parameters of the model are fixed. Because they depend on the strain rate, their identification needs a shear test at a different strain rate.

$$
\dot{\varepsilon}_{\text {roxm }}=\frac{3}{2}\left(\frac{\langle f\rangle}{K}\right)^{n}
$$


The viscoplastic strain rate can finally be written as in Eq.25. In this particular case, in order to reduce the number of parameters, an associative model has been developed, which means that the flow rule is described using the same function as the yield surface [15].

$$
\dot{\varepsilon}_{v p}=\dot{e}_{v c u m} \frac{\partial f}{\partial \underline{\sigma}}
$$

\subsubsection{Identification of viscoplastic parameters using monotonic tests}

The viscoplastic parameters of the model are also determined using an inverse identification method. It is important to note that the viscoelastic part is enabled during this operation.

a) Identification of the elastic limit: $R_{0}, a_{1}$

The elastic threshold $R_{0}$ is calculated at the end of the linear part of the mechanical behaviour. Because of the definition of the yield surface (Eq.22), only two monotonic tests, performed in tensile $\left(\gamma=0^{\circ}\right)$ and shear $\left(\gamma=90^{\circ}\right)$ loading, are needed to obtain the experimental yield surface (see the points in Fig.15a). These values of the elastic threshold are then used to perform a numerical simulation of the two loading conditions. For each case, the stress tensor in the middle of the adhesive joint, is plotted in a von Mises vs hydrostatic pressure plane [24]. It is clear that for $\gamma=90^{\circ}$, no hydrostatic pressure is obtained in the middle of the joint. However, for tensile behaviour, due to the complex state of the stress in the bonded joint, the same point, in the middle of the joint is characterised by a hydrostatic and a deviatoric stress. The yield function is then analytically identified. The slope of this function allows the determination of the $a_{1}$ parameter (Fig.15a).

b) Identification of the hardening: $H, Q, b$

The hardening parameters are also identified using the inverse identification method. The $0.2 \mathrm{kN} / \mathrm{s}$ monotonic shear test (Fig.15b) is used for the parameters identification. An 
optimization between the experimental curve and the numerical response of the finite element model has been performed in order to minimize the error between the two behaviours.

It is important to notice that since the model is not able to predict the hardening observed at the end, the last part of the curves was not considered during the identification process. This complex phenomenon can be explained by the presence of porosities or charges and to be able to model it, other components should be added to the present version of the model.

c) Identification of the viscous parameters: $K, n$

Once the hardening parameters have been determined, the couple $(K, n)$ is identified. Because they depend on the strain rate, a second monotonic shear test $(2 \mathrm{kN} / \mathrm{s})$ is used for the identification (Fig.15b).

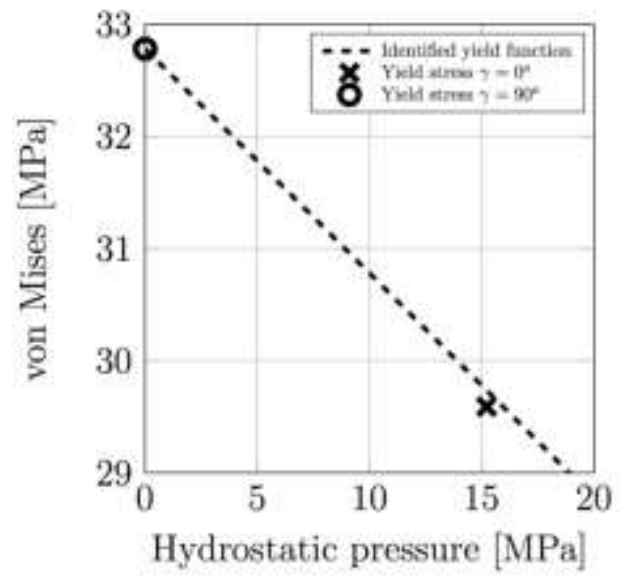

(a)

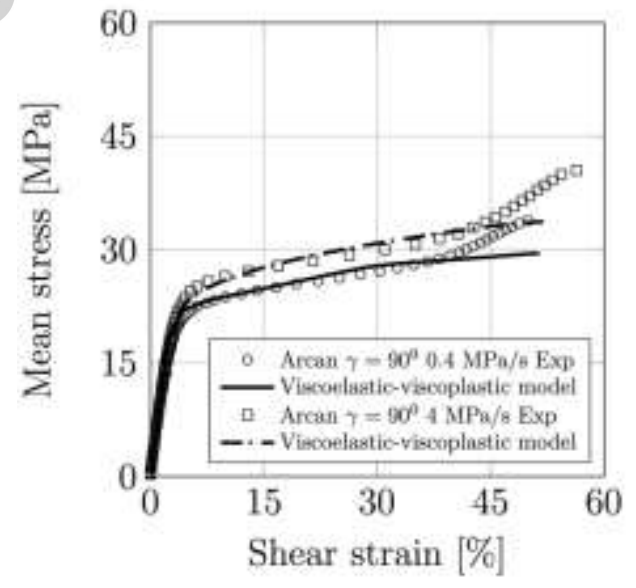

(b)

Figure 15: (a) Experimental and numerical yield function (b) Identification of the viscoplastic parameters

It is important to note that in order to identify the viscoplastic behaviour, two monotonic shear tests have been entirely used. The yield surface has been determined using the elastic parts of tensile and combined tensile/shear tests. These tests can be used to validate the non-linear behaviour. The identified parameters can be found in Table 3. 
Table 3: Identified viscoplastic parameters

\begin{tabular}{cccccccc}
\hline Parameters & $\mathrm{R}_{0}$ & $\mathrm{a}_{1}$ & $\mathrm{H}$ & $\mathrm{Q}$ & $\mathrm{b}$ & $\mathrm{n}$ & $\mathrm{K}$ \\
\hline Unit & $\mathrm{MPa}$ & - & $\mathrm{MPa}$ & $\mathrm{MPa}$ & - & - & - \\
Value & 32 & 0.2 & 2 & 2.5 & 45 & 3.5 & 10
\end{tabular}

\subsection{Validation of the viscoelastic-viscoplastic model}

The model validation is based on the comparison between experimental results and numerical predictions. In order to do this, experimental data that have not been used in the identification procedure are analysed.

The first test used to validate the model is a combined tensile/shear monotonic test. The positioning of the Arcan device can be observed in Fig.1a, where $\gamma=45^{\circ}$. The load and the displacements are decomposed into a normal component and a tangential one. The prediction of the model and the experimental curves are compared in Fig.16a,16b. As it can be observed, the obtained results are very satisfying. Moreover, this test was also used to validate the yield function. The elastic threshold for this type of test was calculated as it has been presented in section 3.3.2.a). The representation of the yield stress for the combined tensile/shear test $\left(\gamma=45^{\circ}\right)$ in Fig.17a indicates that the yield function is correctly predicting the evolution of the yield stress.

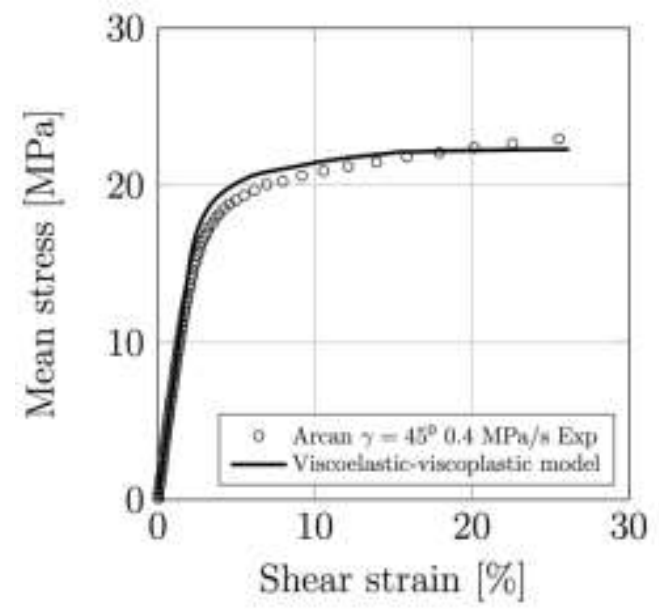

(a)

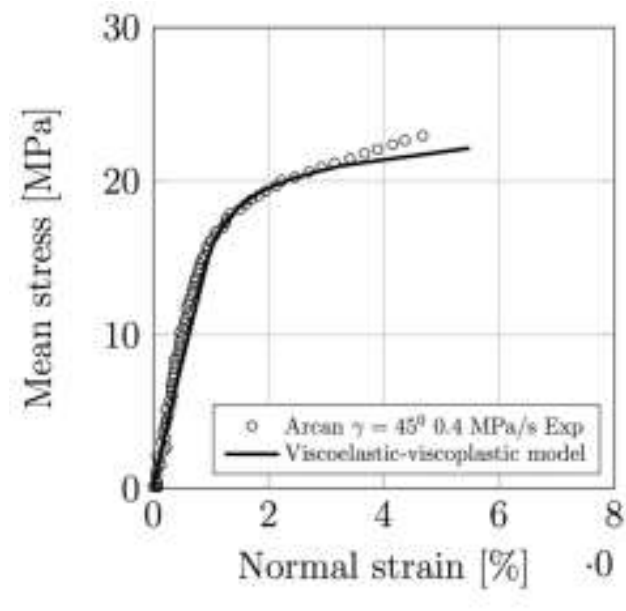

(b)

Figure 16: Viscoelastic-viscoplastic model validation in combined tensile/shear test 


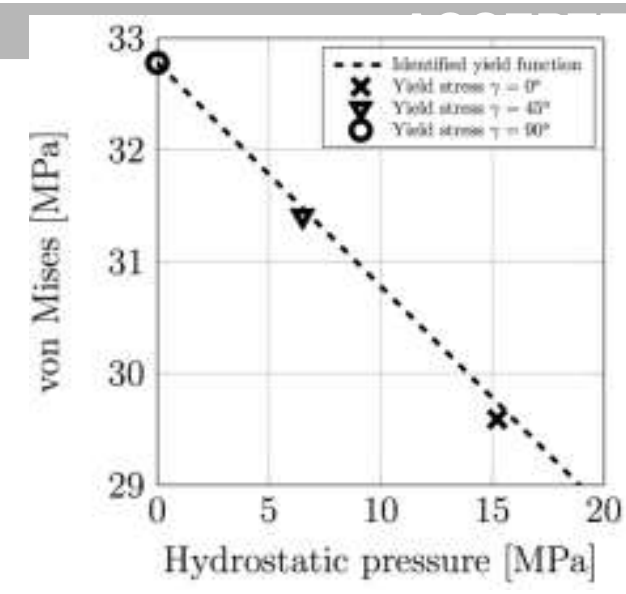

(a)

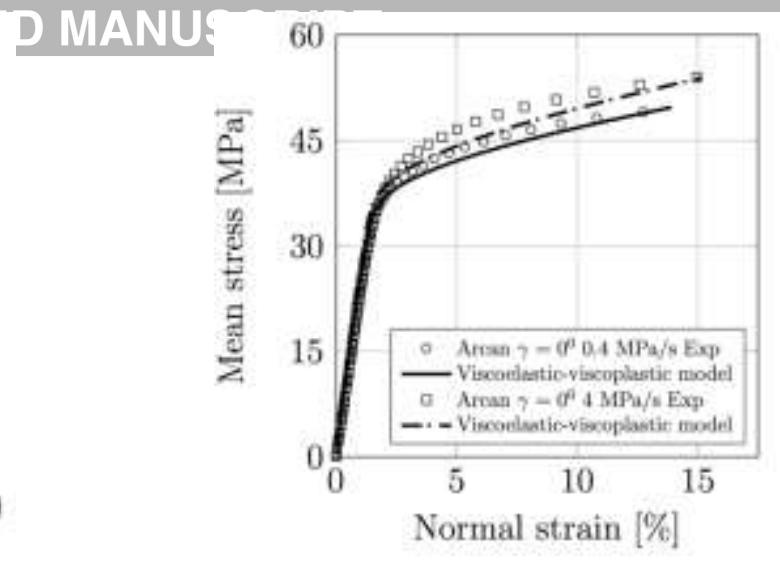

(b)

Figure 17: (a) Validation of the yield function; (b) Strain rate effect validation in monotonic tensile tests

The prediction of the strain rate effects was validated using a monotonic tensile test at $2 \mathrm{kN} / \mathrm{s}$ (Fig.17b). The elastic modulus does not depend on the loading rate for this type of adhesive, instead, the failure load is increasing. It can be observed that by multiplying the loading rate by ten, the failure load is increased by about ten percent. This change in the mechanical behaviour of the adhesive can be predicted by the presented model.

For both tensile and shear creep behaviour, multi-level recovery tests were also used to validate the model. As it can be observed in Fig.18, in the two cases, even though the model has been identified on the creep levels it can predict the behaviour of the recovery levels. An interesting point that should be mentioned is that looking at the last recovery level in shear(Fig.18a) the viscoelastic strain is not totally recovered when completely unloading the bonded joint. The prediction of this recovery level is still very accurate. Regarding the tensile recovery levels(Fig.18b), the model is a little bit less accurate but this can be explained by the very small displacements that are measured. 


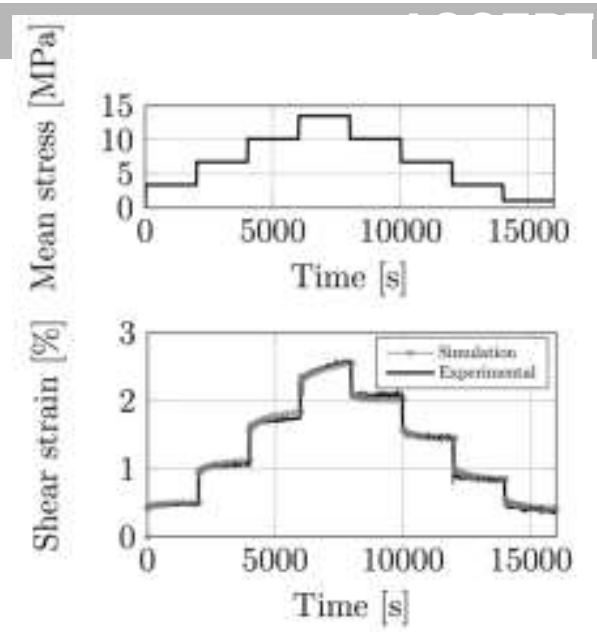

(a)

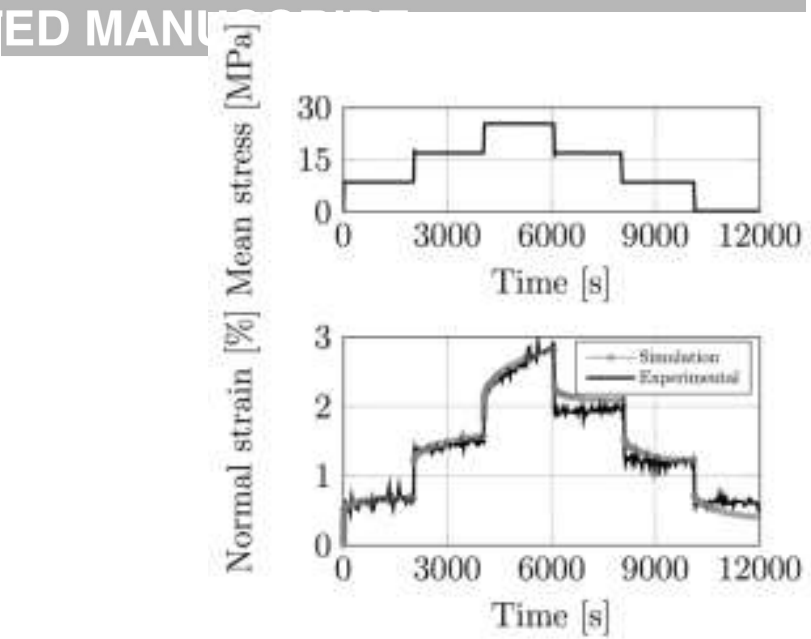

(b)

Figure 18: Model validation in multi-level creep/relaxation (a) shear and (b) tensile tests

\section{Conclusion and perspectives}

This paper presents an original development of a viscoelastic-viscoplastic model capable of describing the mechanical behaviour of an adhesive in a bonded joint. As it can be seen in literature, the mechanical behaviour of some types of adhesives can be modeled only by using a viscoelastic constitutive law. In the case studied here, viscoelasticity was not sufficient to describe the important non-linearity in the behaviour of the studied adhesive. Moreover, the viscoelastic spectral model, initially developed to describe the composites matrix mechanical behaviour and then adapted by Badulescu et al. [11] for adhesives, has been modified in order to take into account the effect of the von Mises and the hydrostatic stress as proposed by Carrere et al. [26].

For identification and validation of the viscoelastic part of the model, only one multilevel creep recovery test is needed in two directions (tensile and shear). The loading of each level should be below the elastic threshold. For a better understanding of the timedependent behaviour, long creep levels are recommended. The creep levels are only used for identification of the model parameters. The validation of the model is made afterwards using the recovery levels of the same sample.

Once the viscoelastic part of the model is defined, the viscoplastic part of the model can be identified. The pseudo yield surface is determined using monotonic tests in three 
directions $\left(\gamma=0^{0}, \gamma=45^{0}, \gamma=90^{\circ}\right)$. For the identification of the loading rate dependent parameters $K$ and $n$, a second monotonic shear test $\left(\gamma=90^{\circ}\right)$, performed at another loading rate was needed.

Even though the proposed model has an important number of parameters (10 for the viscoelastic part and 7 for viscoplasticity), only two creep tests and three monotonic tests are needed to identify all these parameters. It is also important to highlight the method of identification which is simple to implement. Each parameter is determined step by step using the inverse identification method.

For further investigations, the sensibility of each parameter of the model can be studied. In order to quantify it, first, the relevant tests should be defined for each type of parameters (viscoelastic or viscoplastic). In a second phase, an approach should be developed to calculate an error with respect to the global mechanical response after changing a specific parameter with a certain ratio.

As from a qualitatively point of view, it can be confirmed that the viscoelastic parameters $\left(a_{i}, d^{\max }{ }_{i}, y_{i}{ }^{0}, y_{i}^{C}\right.$ where $\left.i=\{H, D\}\right)$ have a great influence in creep behaviour but they don't seem to influence that much the global mechanical response in monotonic tests. The spectrum parameters $\left(n_{0}, n_{c}\right)$ characterize the form of the creep behaviour but they will not impact the predicted strains. Viscoplastic parameters have great influence in both shear and tensile monotonic behaviour but they will not influence the creep behaviour as long as the applied load will be below the elastic limit.

Results show that a good agreement with the experimental curves is obtained. To test its capability, it would be interesting to test the prediction of the model in more realistic joints such as single lap joints. In further investigations, the model can be used to predict the cyclic mechanical behaviour, which is very important when considering fatigue lifetime prediction. Another aspect is to identify the evolution of the model parameters as a function of water ageing of the material, in order to predict the influence of water on the mechanical behaviour. 


\section{References}

[1] F. X. Irisarri, F. Laurin, N. Carrere, and J-F. Maire. Progressive damage and failure of mechanically fastened joints in CFRP laminates - part i: Refined finite element modelling of single-fastener joints. Composite Structures, 94(8):2269-2277.

[2] Ochsner A. da Silva L.F.M. and R.D. Adams. Handbook of adhesion technology, Ed. Springer, 2011.

[3] G. C. Papanicolaou, P. Charitidis, D. E. Mouzakis, E. Karachalios, G. Jiga, and D. V. Portan. Experimental and numerical investigation of balanced boron/epoxy single lap joints subjected to salt spray aging. International Journal of Adhesion and Adhesives, 68:9-18, 2016.

[4] J-Y. Cognard, R. Cr'eac'hcadec, L. Sohier, and P. Davies. Analysis of the nonlinear behaviour of adhesives in bonded assemblies-comparison of TAST and arcan tests. International Journal of Adhesion and Adhesives, 28(8):393-404, 2008.

[5] J. Y. Cognard, P. Davies, B. Gineste, and L. Sohier. Development of an improved adhesive test method for composite assembly design. Composites Science and Technology, 65(3):359-368, 2005.

[6] L.F.M. da Silva and A. Ochsner. Modeling of adhesively bonded joints, Ed. Springer, 2008.

[7] T. Iwamoto, T. Nagai, and T. Sawa. Experimental and computational investigations on strain rate sensitivity and deformation behaviour of bulk materials made of epoxy resin structural adhesive. International Journal of Solids and Structures, 47(2):175- 185 .

[8] X. X Yu, A. D Crocombe, and G Richardson. Material modelling for rate-dependent adhesives. International Journal of Adhesion and Adhesives, 21(3):197-210.

[9] J. A. Harris and R. D. Adams. An assessment of the impact performance of bonded joints for use in high energy absorbing structures. Proceedings of the Institution of Mechanical Engineers, Part C: Journal of Mechanical Engineering Science, 199(2):121-131, 1985.

[10] L. F. M. da Silva, R. D. Adams, and M. Gibbs. Manufacture of adhesive joints and bulk specimens with high-temperature adhesives. International Journal of Adhesion and Adhesives, 24(1):69-83, 2004.

[11] C. Badulescu, C. Germain, J-Y. Cognard, and N. Carrere. Characterization and modelling of the viscous behaviour of adhesives using the modified arcan device. Journal of Adhesion Science and Technology, 29(5):443-461, 2015.

[12] A. D. Crocombe. Modelling and predicting the effects of test speed on the strength of joints made with FM73 adhesive. International Journal of Adhesion and Adhesives, 15(1):21-27. 
[13] P. Majda and J. Skrodzewicz. A modified creep model of epoxy adhesive at ambient temperature. International Journal of Adhesion and Adhesives, 29(4):396-404.

[14] G.D. Dean, L.N. McCartney, R. Mera, and J.M. Urquhart. Modeling nonlinear viscoelasticity in polymers for design using finite element analysis. Polymer Engineering and Science, 51(11):2210-2219, 2011.

[15] R. Mahnken and M. Schlimmer. Simulation of strength difference in elasto-plasticity for adhesive materials. International Journal for Numerical Methods in Engineering, 63(10):1461-1477, 2005.

[16] P. Jousset and M. Rachik. Implementation, identification and validation of an elastoplastic-damage model for the finite element simulation of structural bonded joints. International Journal of Adhesion and Adhesives, 50:107-118, 2014.

[17] W. K. Chiu and R. Jones. Unified constitutive model for thermoset adhesive, FM73. International Journal of Adhesion and Adhesives, 15(3):131-136.

[18] P. C. Pandey, H. Shankaragouda, and Arbind Kr. Singh. Nonlinear analysis of adhesively bonded lap joints considering viscoplasticity in adhesives. Computers \& Structures, 70(4):387-413, 1999.

[19] M. Zgoul and A. D. Crocombe. Numerical modelling of lap joints bonded with a ratedependent adhesive. International Journal of Adhesion and Adhesives, 24(4):355-366, 2004.

[20] Claudiu Badulescu, Jean-Yves Cognard, Romain Cr'eac'hcadec, and P Vedrine. Analysis of the low temperature-dependent behaviour of a ductile adhesive under monotonic tensile/compression-shear loads. International Journal of Adhesion and Adhesives, 36:56-64, 2012.

[21] J-Y. Cognard, L. Sohier, and P. Davies. A modified arcan test to analyze the behaviour of composites and their assemblies under out-of-plane loadings. Composites Part A: Applied Science and Manufacturing, 42(1):111-121, 2011.

[22] J-Y. Cognard. Numerical analysis of edge effects in adhesively-bonded assemblies application to the determination of the adhesive behaviour. Computers \& Structures, 86(17):1704-1717, 2008.

[23] F Laurin, N Carrere, and J-F Maire. A multiscale progressive failure approach for composite laminates based on thermodynamical viscoelastic and damage models. Composites Part A: Applied Science and Manufacturing, 38(1):198-209, 2007.

[24] J. Maurice, J-Y Cognard, R. Creac'hcadec, P. Davies, L. Sohier, and S. Mahdi. Characterization and modelling of the $3 \mathrm{~d}$ elastic-plastic behaviour of an adhesively bonded joint under monotonic tension/compression-shear loads: influence of three cure cycles. Journal of Adhesion Science and Technology, 27(2):165-181, 2013.

[25] J-Y Cognard, R. Cr'eac'Hcadec, J. Maurice, P. Davies, M. Peleau, and L. F. M. Da Silva. Analysis of the influence of hydrostatic stress on the behaviour of an adhesive in a 
bonded assembly. Journal of Adhesion Science and Technology, 24(11):1977- 1994, 2010.

[26] N. Carrere, F. Laurin, and J-F. Maire. Micromechanical-based hybrid mesoscopic 3d approach for non-linear progressive failure analysis of composite structures. Journal of Composite Materials, 46(19-20):2389-2415, September 2012. 